\title{
Prediction of Disease Diagnosis by Automate Segmentation Approach in Retinal Images
}

\author{
R. Karthiyayini ${ }^{1}$, Dr. R. Balasubramanian ${ }^{2}$ \\ Research Scholar, Bharathidasan University ${ }^{1}$ \\ Assistant Professor, Department of Computer Applications, Anna University, Tiruchirappalli, India ${ }^{1}$ \\ Professor and Head, P.G \& Research Dept of Computer Science, J.J. College of Arts and Science, Pudukkottai, India ${ }^{2}$
}

\begin{abstract}
Research in the field of medicine suggests that abnormal pressure and glucose levels are a major cause of several critical complaints. A retinal image provides a snapshot of what is happening inside the human body. In particular, the state of the retinal vessels has been shown to reflect the cardiovascular condition of the body. Retinal images provide considerable information on pathological changes caused by local ocular disease which reveals diabetes, hypertension, arteriosclerosis, cardiovascular disease and stroke. Computer-aided analysis of retinal image plays a central role in diagnostic procedures. However, automatic retinal segmentation is complicated by the fact that retinal images are often noisy, poorly contrasted, and the vessel widths can vary from very large to very small. In this paper, we place focus to implement automate segmentation approach based on graph theoretical method. The scope of this paper is to provide blood vessels regional information by the measure of median filter algorithm. We proposed a novel computational approach for the segmented vascular structure as a vessel segment graph and solve the problem to identify vessels as the one of the findings of blood vessels in the graph given a set of constraints such as CRAE and CRVE. These measurements are found to have good correlation with hypertension, coronary heart disease, and stroke. Moreover, these require the accurate extraction of distinct vessels from a retinal image and a classification method is used to reveals the optimization problem and evaluate it on a real-world dataset of retinal images.
\end{abstract}

Keywords: Automatic segmentation, Computational approach, Classification Data mining.

\section{INTRODUCTION}

Application of computational techniques in the field of medicine has been an area of intense research in recent years. Data mining is a technique that mines data effectively to get useful information. Data mining plays a crucial role in mining of healthcare data. Healthcare data can be collected from various hospitals. The assimilated data can be used to analyze the patient reports which help in identifying the patterns present in the database which further helps to get information about various diseases present, their symptoms, causes, remedies and precautions that can helps to prevent the occurrence of various diseases.

[1] Retinal image processing is greatly required in diagnosing and treatment of many diseases affecting the retina and the choroid behind it. Diabetic retinopathy is one of the complications of diabetes mellitus affecting the retina and the choroid. Retinal imaging is a recent technological advancement in eye care. [2]It enables optometrist to capture a digital image of the retina, blood vessels and optic nerve located at the back of eyes. This aids in the early detection and management of diseases that can affect both eyes and overall health. This includes glaucoma, macular degeneration, diabetes and hypertension. With retinal imaging technology, the most subtle changes to the structures at the back of eyes can be detected.
[3] In this condition, a network of small blood vessels, called choroidal neovascularization $(\mathrm{CNV})$, arises in the choroid and taking a portion of the blood supplying the retina. As the amount of blood supplying the retina is decreased, the sight may be degraded and in the severe cases, blindness may occur. [4] The physicians try to treat this dangerous disorder by applying optical energy to photocoagulate the neovascularization. Argon laser is used in photocoagulation purposes to cauterize the small vessels which increases the amount of blood supplying the retina and thus maintaining the sight. This treatment modality is achieved in many sessions. The physician asks the patient to fixate his/her eye to be able to direct the laser beam to the affected area. The current success rate of this procedure is below $50 \%$ for eradication of $\mathrm{CNV}$ following one treatment session with a recurrence and/or persistence rate of about $50 \%$. The latter condition requires repeating the treatment. Each treatment repetition in turn has a 50\% failure rate. Moreover, several studies indicate that incomplete treatment was associated with poorer prognosis than no treatment. Consequently, the need to develop an automated laser system to treat the whole retina in one session has become a necessity. This system is intended to scan the retina and track it applying the laser energy to whole area except the sensitive objects that may be damaged by the laser energy. The system is assumed to do this by capturing the retinal images using a fundus 
camera [5]. These images are to be accurately segmented to extract the sensitive objects in the retina such as the blood vessel tree, the optic disk, the macula and the region between the optic disk and the macula. The positions of laser shots are to be distributed in the rest of the retina. Also a robust registration technique is to be applied to detect the motion parameters of the retina to update the positions of laser shots accordingly. Moreover, the fundus camera can only provide an image for a portion the retina but not the whole retina. The physician sometimes needs to have a complete image for the retina to be able to have a reliable diagnosis and hence plan for right treatment. This problem may be overcome by some image processing algorithms to build a complete map for the retina. The retina is a layered structure with several layers of neurons interconnected by synapses. The only neurons that are directly sensitive to light are the photoreceptor cells. These are mainly of two types: the rods and cones. Rods function mainly in dim light and provide black-and-white vision, while cones support daytime vision and the perception of color. A third, much rarer type of photoreceptor, the intrinsically photosensitive ganglion cell, is important for reflexive responses to bright daylight.

The manuscript is organized as follows. Section 2 focuses on the literature survey in the related field. Section 3 highlights the proposed methodology while section 4 Architecture diagram for computational Approach section 5 presents the experimental results. Section 5 concludes the paper.

\section{LITERATURE REVIEW}

[6] Wang et al, introduced a novel vessel enhancement technique based on the matched filters with multiwavelet kernels (MFMK) and identifies kernels separating vessels from clutter edges and bright, localized features (e.g., lesions). For noise attenuation and vessel localization, authors proposed a multi scale hierarchical decomposition, which is particularly effective for the normalized enhanced image. This process performs an iterative segmentation at increasing image resolutions, locating smaller and smaller vessels. [7] B. Zhang et al, adopted a single scale parameter controls the level of detail included in the vessel map. Then discussed a necessary condition to achieve the optimal decomposition, deriving a rule to identify the optimal number of the hierarchical decomposition. This method does not require preprocessing and training it can therefore be used directly on images with different characteristics. In addition, it relies on adaptive thresholding so that no numerical parameter is tuned manually to obtain a binary mask.

[8] M.M.Fraz et al, analyses Retinal vessel segmentation and delineation of morphological attributes of retinal blood vessels, such as length, width, tortuosity and/or branching pattern and angles are utilized for diagnosis, screening, treatment, and evaluation of various cardiovascular and ophthalmologic diseases such as diabetes, hypertension, arteriosclerosis and chorodial neovascularization. This survey is unique in that it particularly focuses on the algorithms and methodologies for the segmentation of blood vessels in two dimensional colored retinal images acquired from a fundus camera and no such review exists to the best of our knowledge. [9] $\mathbf{M}$. Palomera et al, discussed the objectives of this paper are to review the retinal vessel segmentation methodologies; to provide a detailed resource of the algorithms employed for vessel segmentation to researchers for ready reference; to discuss the advantages and disadvantages of the various approaches; to discuss the current trends and future directions and summarize the open problems.

[10] K. Sun and S. Jiang proposed an active contour model for automatic vascular segmentation on 2-D angiogram, where the local morphology feature is integrated into the region-based deformable model. Compared to LBF, the proposed method applies directional measure substituting isotropic one in data fitting term. Different fitting formulation, maximum fuzzy opening, and minimum fuzzy opening are used for object and background, respectively. They can be computed before evolution without need to update in each contour evolution. Therefore, the proposed method achieves a robust evolution of level set against initial condition. [11] G. Lathen et al, used the scale in local image information extraction is detected with an adaptive process instead of predefined ones adopted in LBF. In the proposed method, the local region associated to curve is also used to confine the energy of level set.

Thus the existing work first primarily focused on the retinal microvasculature shares anatomical and physiological characteristics with the vessel structure in other parts of the human body. Some imaging techniques, such as retinographies, provide non invasive views of the blood vessels in the retina. In this sense, numerous studies have reported that retinal micro vascular abnormalities, such as arteriovenous nicking, focal or generalized arteriolar narrowing and venular dilation, are related to different pathologies, such as hypertension and diabetes. The arterio-venous ratio (AVR), this is, the ratio between arteriolar and venular diameters, is a measure often used as a marker for several diseases. [12]A large number of works on general vessel segmentation have appeared in the literature. However, the automated classification of the segmented vasculature in arteries and veins has received limited attention. A semi-automatic method for the analysis of retinal vascular trees in which the venous and arterial trees were analyzed separately was presented. A more recent work shows a method to label all vessels as either artery or vein using existing vessel segmentation and some manually labeled starting vessel segments. The work closest to this one is an automated classification method in which the vasculature is segmented using a vessel tracking procedure and the vessel centerlines are detected. After defining an area of interest around the optic disc and dividing this area into four quadrants, color based features are extracted from the vessel segments that are then classified into arteries and veins using an unsupervised clustering method. 
Vol. 5, Issue 12, December 2016

\section{PROPOSED METHODOLOGY}

Examination of blood vessels in the eye allows detection of eye diseases such as glaucoma and diabetic retinopathy. Traditionally, the vascular network is mapped by hand in a time-consuming process that requires both training and skill. Automating the process allows consistency, and most importantly, frees up the time that a skilled technician or doctor would normally use for manual screening. In this paper, we proposed to implement automatic process to examine the blood vessels to identify the cardio vascular diseases in retinal images. The proposed method utilizes the concept of active contours to remove noise, enhance the image, track the edges of the vessels, calculate the perimeter of vessels and identify the cardio diseases. We intended to implement graph theoretical model to segment blood vessels and calculate perimeter of the blood vessels. We adopted an efficient and effective infinite perimeter active contour model with hybrid region terms for vessel segmentation with good performance. This will be a powerful tool for analyzing vasculature for better management of a wide spectrum of vascular-related diseases. Retinal vascular caliber (CRAE and CRVE) was analyzed as continuous variables. We used analysis of covariance to estimate mean retinal vascular caliber associated with the presence versus absence of categorical variables or increasing quartiles of continuous variables to predict the cardio vascular diseases.

The automatic analysis of retinal fundus, a number of algorithms have been proposed for extracting the vascular structure and for identifying non-vascular lesion (exudates, haemorrhages, ischemic regions). The first changes in the retina that point out the onset of a retinopathy, e.g. from a systemic disease, appear in the vessels. Changes in vessel structure can affect very differently arteries and veins. In order to realize an automatic tool for the diagnosis and grading of retinopathy, it is mandatory to automatically distinguish between arterial and venous vessels (A/V classification). The presence of inter- and intra-image contrast, luminosity and color variability is a first problem, whereas the fading of the differences between the two types of vessels in the periphery of the retina is a second, but of no lesser impact, problem. Even after image contrast and luminosity normalization, $\mathrm{A} / \mathrm{V}$ may be recognized reasonably well only in an area around the optic disc: inside the optic disc, vessels become so intertwined that it is very difficult even for an expert to track a vessel, whereas in the periphery of the image (far from the optic disc) they become thinner and thinner and almost undistinguishable. Moreover, even around the optic disc only vessels close to each other can be reliably recognized as arteries or veins by direct comparison, whereas vessels far apart from each other can be easily misclassified without any further knowledge other than their image features. The retinal vessel network has a specific structure: the main vessels emerge at the optic disc and then follow a double-parabolic path by branching and thinning. This structure applies to both arteries and veins and we can assume that, at a small distance from the optic disc border, both types of vessels are distributed in a balanced way around the optic disc. These observations led us in our strategy to develop a reliable $\mathrm{A} / \mathrm{V}$ classification technique. To begin with, we decided to classify the vessels only in a well defined concentric zone around the optic disc. Then, by using the vessel structure reconstructed by tracking techniques, we would propagate this classification outside this zone, where little or no information is available to discriminate arteries from veins. Second, the A/V classification algorithm would not be designed considering all together the vessels in the zone, but rather partitioning the zone in four quadrants and working separately and locally on each of them.

The following phases are developed in the methodology to identified retinal based disease diagnosis:

- Retinal image acquisition

- Preprocessing

- IPACHI model

- Vessel classification

- Disease diagnosis

A. Retinal image acquisition

Retinal images of human plays an important role in the detection and diagnosis of cardio vascular diseases that including stroke, diabetes, arteriosclerosis, cardiovascular diseases and hypertension, to name only the most obvious diseases. Vascular diseases are often life-critical for individuals, and present a challenging public health problem for society. Therefore, the detection for retinal images is necessary, and among them the detection of blood vessels is most important. The alterations about blood vessels, such as length, width and branching pattern, can not only provide information on pathological changes but can also help to grade diseases severity or automatically diagnose the diseases. In this phase, we upload the retinal images. The fundus of the eye is the interior surface of the eye, opposite the lens, and includes the retina, optic disc, macula and fovea, and posterior pole. The fundus can be examined by ophthalmoscopy and/or fundus photography. The retina is a layered structure with several layers of neurons interconnected by synapses. In retina we can identify the vessels. Blood vessels show abnormalities at early stages also blood vessel alterations. Generalized arteriolar and venular narrowing which is related to the higher blood pressure levels, which is generally expressed by the Arteriolar-toVenular diameter ratio. In this work, we have constructed a dataset of images for the training and evaluation of our proposed method. This image dataset was acquired from publically available datasets such as DRIVE and STAR. Each image was captured using 24 bit per pixel (standard RGB) at $760 \times 570$ pixels. First, proposed method has only been tested against normal images which are easier to distinguish. Second, some level of success with abnormal vessel appearances must be established to recommend clinical usage. As can be seen, a normal image consists of blood vessels, optic disc, fovea and the background, but the abnormal image also has multiple artifacts of distinct 
shapes and colors caused by different diseases. In fig (a) is denoted as normal image and (b) is denoted as diseased image.
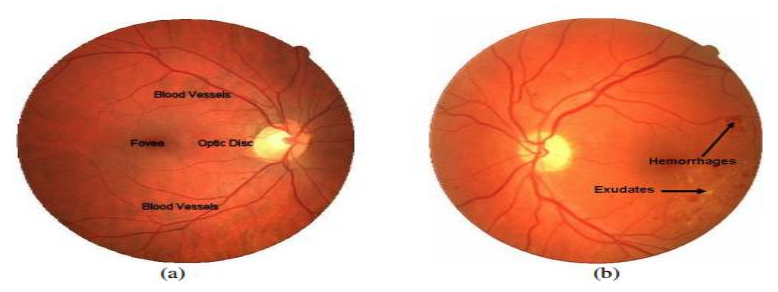

\section{B. Preprocessing}

In this phase, we perform the gray scale conversion operation to identify black and white illumination. Noise in colored retinal image is normally due to noise pixels and pixels whose color is distorted so implement sharpening filter can be used to enhance and sharpen the vascular pattern for preprocessing and blood vessel segmentation of retinal images performing well in preprocessing, enhancing and segmenting the retinal image and vascular patter.

Human perception is highly sensitive to edges and fine details of an image, and since they are composed primarily by high frequency components, the visual quality of an image can be enormously degraded if the high frequencies are attenuated or completed removed. In contrast, enhancing the high-frequency components of an image leads to an improvement in the visual quality. Image sharpening refers to any enhancement technique that highlights edges and fine details in an image. Image sharpening is widely used in printing and photographic industries for increasing the local contrast and sharpening the images. In principle, image sharpening consists of adding to the original image a signal that is proportional to a high-pass filtered version of the original image. In this filter, the original image is first filtered by a high-pass filter that extracts the high-frequency components, and then a scaled version of the high-pass filter output is added to the original image, thus producing a sharpened image of the original. Note that the homogeneous regions of the signal, i.e., where the signal is constant, remain unchanged.

\section{Vessel segmentation}

In this phase, we can perform feature extraction and vessel segmentation steps using graph theoretical model. It can create vascular network using active contour with nearest neighbor measure with neighborhood function. We can extract the map is a representation of the vascular network, where each node denotes an intersection point in the vascular tree, and each link corresponds to a vessel segment between two intersection points. For generating the graph, we have used active contour method. The nodes are extracted from the centerline image by finding the bifurcation points which are detected by considering pixels with more than two neighbors and the endpoints or terminal points by pixels having just one neighbor. In order to find the links between nodes (vessel segments), all the bifurcation points and their neighbors are removed from the centerline image and as a result we get an image with separate components which are the vessel segments. Vessels segmentation binary mask is created by detecting vessels edges from sharpened image. The blood vessels are marked by the masking procedure which assigns one to all those pixels which belong to blood vessels and zero to non vessels pixels. Final refined vessel segmentation mask is created by active contour model. Active contour model, also called snakes, is a framework in computer vision for delineating an object outline from a possibly noisy 2D image. A snake is an energy minimizing, deformable spline influenced by constraint and image forces that pull it towards object contours and internal forces that resist deformation. Snakes may be understood as a special case of the general technique of matching a deformable model to an image by means of energy minimization. In two dimensions, the active shape model represents a discrete version of this approach, taking advantage of the point distribution model to restrict the shape range to an explicit domain learned from a training set. It provides the segmentation mask for preprocessed retinal images.

\section{Vessel classification}

The segmented vessels are classified into arteries and veins. Correct classification of vessels is vital, because heart diseases affect arteries and veins differently. The alterations in veins and arteries cannot be analyzed without distinguishing them. After extraction of blood vessels, feature vector is formed based on properties of artery and veins. The features get extracted on the basis of centerline extracted image and a label is assigned to each centerline, indicating the artery and vein pixel. Based on these labelling phase, the final goal is now to assign one of the labels with the artery class (A), and the other with vein class (V). In order to allow the final classification between $\mathrm{A} / \mathrm{V}$ classes along with vessel intensity information the structural information and are also used.

This can be done using SVM classification. The trained classifier is used for assigning the $\mathrm{A} / \mathrm{V}$ classes to each one of the sub graph labels. First, each centerline pixel is classified into A or $\mathrm{V}$ classes, then for each label ( $\mathrm{Ci} \mathrm{j} \mathrm{,} \mathrm{j} \mathrm{=}$ 1,2 ) in sub graph $i$, the probability of its being an artery is calculated based on the number of associated centerline pixels classified by LDA to be an artery or a vein. The probability of label $\mathrm{Ci} \mathrm{j}$ to be an artery is

$$
\mathrm{Pa}(\mathrm{Ci} \mathrm{j})=\text { na } \mathrm{Ci} \mathrm{j} /(\text { na } \mathrm{Ci} \mathrm{j}+\text { nvCi })
$$

Where na $\mathrm{Ci} \mathrm{j}$ is the number of centerline pixels of a label classified as an artery and $\mathrm{nv} \mathrm{Ci} \mathrm{j}$ is the number of centerline pixels classified as a vein. For each pair of labels in each sub graph, the label with higher artery probability will be assigned as an artery class, and the other as a vein class. Finally, to prevent a wrong classification as a result of a wrong graph analysis, we calculate the probability of being an artery or a vein for each link individually. 
D. Disease diagnosis

In this phase, we can diagnosis the diseases using AVR ratio based on CRAE and CRVE measurements. The vessel measurements CRAE, CRVE have been found to be correlated with risks factors of cardiovascular diseases and are positive real numbers. The major systemic determinant for smaller CRAE is higher blood pressure whereas wider CRVE is mainly due to current cigarette smoking, higher blood pressure, systemic inflammation and obesity. Those with higher blood pressure (75th percentile) had on average 4.8 microns smaller CRAE and 2.6 microns wider CRVE than those with lower blood pressure (25th percentile). A more recent study found a strong negative correlation between renal function and retinal parameters (CRAE and CRVE) in a cohort of eighty healthy individuals which suggests a common determinant in preclinical target organ damage. This is in support of earlier studies examining the association between retinal vascular signs and incident hypertension providing evidence that a decrease in CRAE is indeed an antecedent to clinical onset of hypertension and occurs prior to other signs of target organ damage. Besides the value of CRAE in predicting hypertension, it also shows great potential in other pathologies including stroke and diabetes. Generalized arteriolar narrowing as reflected by a decrease in CRAE is associated with an increased risk of stroke with odds ratios reported between 1.1 and 3.0. 15,22,23 While in diabetes, an increase of CRVE is associated with increased incidence of diabetic retinopathy (DR), progression of DR, progression to proliferative DR .

\section{CONCLUSION PROPOSED ARCHITECTURE OF COMPUTATIONAL APPROACH FOR DISEASE PREDICTION}

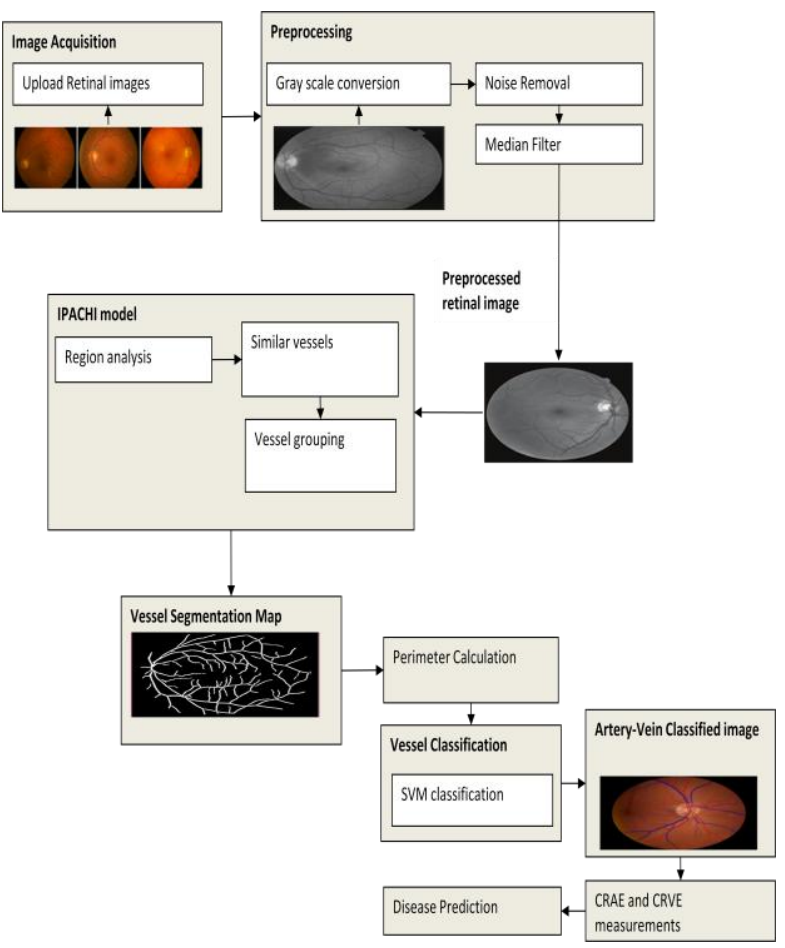

\section{EXPERIMENTAL RESULT ANALYSIS}

The proposed algorithm is implemented in MATLAB environment. The proposed system was constructed and tested using DRIVE datasets. Those databases are divided into training and evaluation dataset to assess the performance of the proposed method. First, fundus images are preprocessed in order to improve image contract. Second, Image segmentation is applied in order to extract features that contain vessel width. Those features are the preliminary signs of cardio vascular diagnosis, a major cause of heart failures. In classification systems, accuracy is often used to evaluate the systems. Accuracy is the overall correctness of the system and is calculated as the sum of correct classifications divided by the total number of classifications.

Accuracy $=$ classified Retina images/Total number of retina images

It is found that the system detects classes successfully with accuracy of $85 \%$.

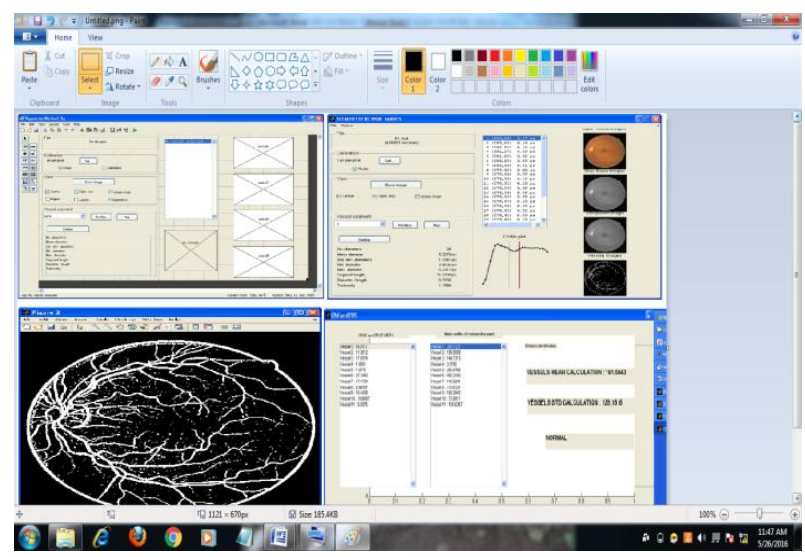

\section{CONCLUSION}

Data mining techniques have been generously applied in the field of medical diagnosis and prognosis in the past. This work concludes that, our system implemented successfully with accurate identification of true vessels to obtain correct retinal ophthalmology measurements. And we implement the post processing step to vessel segmentation. This step is used to track all true vessels and find the optimal forest. We can overcome wrong diagnosis of crossovers by using simultaneous identification of blood vessels from retina. The final goal of the proposed method is to make easier the early detection of diseases related to the blood vessels of retina. Its main advantage is the full automation of the algorithm since it does not require any intervention by clinicians, which releases necessary resources (specialists) and reduces the consultation time, hence its use in primary care is facilitated. Then we realized the classification of arteries and veins in retinal images are essential for the automatic assessment of vascular changes. The graph theoretical 
method with SVM outperforms the accuracy of the SVM classifier by means of intensity features, which shows the significance of using structural information for $\mathrm{A} / \mathrm{V}$ classification. We believe implementation of the proposed system will certainly serve as an aid in assisting ophthalmologists in detecting retinal defects and disease in a timely and precise manner.

\section{REFERENCES}

[1] Imran Quereshi "Glaucoma detection in retinal images using image processing techniques- A survey", International Journal of Advanced Networking and Applications, Volume 7, Issue:2, pp: 2705-2718,2015.

[2] C. Li, C. Xu, C. Gui, and M. Fox, "Distance regularized level set evolution and its application to image segmentation," IEEE Trans. Image Process., vol. 19, pp. 3243-3254, 2010.

[3] Veena Chandran, Dinesh Kumar A, Geetha P.K, Nidhya R,"Deep learning neural network with semi supervised segmentation for predicting retinal and cancer cell diseased images." International journal of advanced research in computer engineering and technology, vol 3, issue 4, April 2014.

[4] A. Perez-Rovira, K. Zutis, J. Hubschman, and E. Trucco, "Improving vessel segmentation in ultra-wide field-of-view retinal fluorescein angiograms," in Proc. IEEE Eng. Med. Biol. Soc., 2011, pp. 2614-2617.

[5] J. Orlando and M. Blaschko, "Learning fully-connected CRFs for blood vessel segmentation in retinal images," in Med. Image Comput. Comput. Assist. Interv., 2014, pp. 634-641.

[6] Y. Wang, G. Ji, P. Lin, and E. Trucco, "Retinal vessel segmentation using multiwavelet kernels and multiscale hierarchical decomposition," Pattern Recogn., vol. 46, pp. 2117-2133, 2013.

[7] B. Zhang, L. Zhang, L. Zhang, and F. Karray, "Retinal vessel extraction by matched filter with first-order derivative of Gaussian," Comput. Biol. Med., vol. 40, pp. 438-445, 2010

[8] M. M. Fraz, P. Remagnino, A. Hoppe, B. Uyyanonvara, A. R. Rudnicka, C. G. Owen, and S. A. Barman, "Blood vessel segmentation methodologies in retinal images - a survey," Comput. Meth. Prog. Bio., vol. 108, pp. 407-433, 2012.

[9] M. Palomera-Prez, M. Martinez-Perez, H. Bentez-Prez, and J. Ortega- Arjona, "Parallel multiscale feature extraction and region growing: application in retinal blood vessel detection," IEEE Trans. Inf. Technol. Biomed., vol. 14, pp. 500-506, 2010.

[10] K. Sun and S. Jiang, "Local morphology fitting active contour for automatic vascular segmentation," IEEE Trans. Biomed. Eng., vol. 59, pp. 464-473, 2012

[11] G. Lathen, J. Jonasson, and M. Borga, "Blood vessel segmentation using multi-scale quadrature filtering," Pattern Recogn. Lett., vol. 31, pp. 762-767, 2010.

[12] C. Lupascu, D. Tegolo, and E. Trucco, "FABC: Retinal vessel segmentation using AdaBoost," IEEE Trans. Inf. Technol. Biomed., vol. 14 , pp. 1267-1274, 2010 . 